\author{
Markus Werthebach, Daniel Sodenkamp, Klaus-Helmut \\ Schmidt
}

\author{
Merkmale, Bedingungen und Folgen von Rollenklarheit bei \\ der Arbeit - ein Vergleich zwischen Leiharbeitnehmern und \\ Mitarbeitern der Stammbelegschaft eines Produktionsbetriebes
}

\begin{abstract}
Anhand eines Praxisbeispiels aus der chemischen Industrie werden die Gründe für den Einsatz von Leiharbeitnehmern sowie deren Einsatzprobleme behandelt. Verglichen wird die Arbeitssituation von 48 Leiharbeitnehmern mit der von 85 Arbeitnehmern der Stammbelegschaft des Entleihunternehmens. Die Ergebnisse zeigen, dass die Leihkräfte ebenso gut instruiert, mit Arbeitsmitteln versehen und mit Leistungsrückmeldung versorgt werden wie die festen Mitarbeiter im Entleihunternehmen. Die Aufgabenklarheit der Leihkräfte entspricht der Aufgabenklarheit der festen Mitarbeiter mit bis zu zwölf Monaten Betriebserfahrung. Eine gemeinsame Betrachtung beider Personengruppen zeigt, dass die Rollenklarheit der Beschäftigten mit zunehmender Betriebserfahrung ansteigt. Weitere Bedingungen von Rollenklarheit liegen im Ausmaß und der Qualität der Informationsversorgung und Leistungsrückmeldung für den Beschäftigten und seine Arbeitsgruppe. Als Folgen einer ausgeprägten Rollenklarheit wurde ein gutes fachliches Selbstvertrauen ermittelt.
\end{abstract}

\title{
1 Problemstellung
}

Ein Leiharbeitsverhältnis ist gegeben, wenn ein selbständiger Unternehmer (Verleiher) einen Arbeitnehmer einstellt und diesen mit dessen Zustimmung zur vorübergehenden oder dauernden Erbringung von Arbeitsleistung einem Dritten (Entleiher) überlässt. Leiharbeitnehmer werden vor allem eingesetzt, um eine hohe technisch-organisatorische Flexibilität in der Produktion bzw. im Angebot von Dienstleistungen gewährleisten zu können. Im einzelnen erlaubt der Einsatz entsprechend qualifizierter Leiharbeitnehmer die Einhaltung zugesagter Lieferzeiten, die Annahme zusätzlicher bzw. größerer Aufträge, den Ausgleich von Personalengpässen (bei kurzfristig erhöhtem Auftragsvolumen bzw. kurzfristig reduzierter Personalkapazität), die rasche Beschaffung von Arbeitskräften ohne zusätzlichen Verwaltungsaufwand sowie die schnelle Freistellung dieser Arbeitskräfte bei Bedarfsende. Darüber hinaus erlaubt sie die Vergabe von Aufgaben, für welche die Stammbelegschaft nicht (bei Andersartigkeit), zu gering (bei Spezialkenntnissen) oder zu hoch (bei Hilfsarbeiten) qualifiziert ist, die Reduzierung von eigenen Personalreserven (Leihpersonal als außerbetriebliche Reserve), die Einsparung von Qualifizierungs- und Weiterbildungsmaßnahmen bei einem 
Einsatz entsprechend spezialisierter Leiharbeitnehmer sowie die Reduzierung des Personalverwaltungsaufwandes.

Daneben kommen arbeitsrechtliche Gründe für den Einsatz von Leiharbeitnehmern zum Tragen:

- Kein Kündigungsschutz und kein bezahlter Urlaub

- Keine Entgeltfortzahlung im Krankheitsfall und an Feiertagen

- Keine Ansprüche auf tariflich geregelte oder betriebsübliche Sonderzahlungen wie zusätzliches Urlaubsgeld, Gratifikationen, Zuschüsse zum Krankengeld

- Unterschreitung kopfzahlbezogener Richtzahlen, z.B. zur Freistellung von Betriebsratsmitgliedern

- Entleihunternehmen haben Anspruch auf eine Ersatzkraft im Krankheitsfall oder bei nicht ausreichender Leistung; das Auswahl- und Ausfallrisiko entfällt für das Entleihunternehmen, da Leiharbeitnehmer, die erkranken oder nicht die erwartete Leistung erbringen, problemlos an das Entleihunternehmen zurückgeschickt werden können.

Zum Stichtag 30.6.1999 waren in der Bundesrepublik Deutschland 286.394 Leiharbeitnehmer beschäftigt, darunter 243.390 Beschäftigte in reinen Zeitarbeitsunternehmen (plus 13 Prozent gegenüber 6/98). Im Jahresverlauf 1999 waren etwa 633.000 Mitarbeiter zumindest zeitweise als Leiharbeitnehmer beschäftigt (plus 10 Prozent gegenüber 1998). Neu eingestellt haben Zeitarbeitunternehmen 1999 etwa 413.000 Mitarbeiter. Davon waren ca. 65 Prozent vorher ohne Beschäftigung. Die Übernahmequote von Leiharbeitnehmern durch die Kundenbetriebe liegt in den letzten Jahren bei etwa 30 Prozent, 1999 waren dies ca. 190.000 Leiharbeitnehmer (Pressemitteilungen des Bundesverbandes Zeitarbeit unter www.bza.de). Für Leiharbeit eignen sich vor allem solche Tätigkeiten, bei denen auf konkrete Einzelanweisungen verzichtet werden kann, weil die Arbeit einfach strukturiert oder aber in Form schriftlicher Arbeitsaufträge bzw. technischer Zeichnungen genau dokumentiert ist (z.B. Baugewerbe, Anlagenbau, Wartungs- und Revisionsarbeiten, Recycling, Abbruch- und Räumarbeiten, Lager- und Verladearbeiten, Straßenbau, mobiles Handwerk, Messebau, Sekretariatsarbeiten, Gastronomie, Landwirtschaft, Gartenbau und Forstwirtschaft, Reinigungsgewerbe). Unter den Leiharbeitnehmern überwiegen die Facharbeiter für das Metallund Elektrogewerbe mit 36,5 Prozent, gefolgt von Hilfspersonal mit 26,4 Prozent, Verwaltungs- und Bürofachkräften mit 12,4 Prozent und Dienstleistungspersonal mit 11,2 Prozent. Techniker sind mit 3,5 Prozent unterrepräsentiert (Stichtag 30.6.1999).

$\mathrm{Zu}$ den häufig erwarteten Problemen beim Einsatz von Facharbeitern bzw. ungelernten Kräften als Leiharbeitnehmer zählen vor allem organisatorische und soziale Eingliederungsprobleme:

- Leiharbeitnehmer werden häufig unzureichend eingewiesen hinsichtlich ihrer Aufgaben und Arbeitsziele, der Zeitvorgaben, der betrieblichen Arbeitsabläufe, der Zuständigkeiten der jeweiligen Vorgesetzten sowie der Gefahrenpunkte und Gefahrstoffe im Betrieb bzw. auf der Baustelle. Leiharbeitern ist darum häufig nicht hinreichend klar, welche Leistungen von ihnen erwartet werden, wie sie ihre Arbeit einteilen und welchen Personen sie zuarbeiten sollen (Rollenklarheit).

- Leiharbeitnehmer werden von der Stammbelegschaft häufig nicht akzeptiert. Zu den gängigen Vorurteilen zählen, Leiharbeitnehmer verfügten über eine mindere Qualifikation, fehlende Betriebserfahrung, unzureichende Arbeitsmotivation sowie ein unzureichendes Commitment gegenüber dem Entleihbetrieb und darum weniger Sorgfalt im Umgang mit Werkstücken, Werkzeugen und Maschinen. 
- Auf der anderen Seite geht der Einsatz von Leiharbeitnehmern mit einem erhöhten Leistungsdruck und einer Beweispflicht für die Stammbelegschaft des Entleihunternehmens einher.

Vorrangige Probleme der Leiharbeitnehmer sind das gesundheitliche Risiko (aufgrund häufig unzureichenden Arbeitsschutzes), das Beschäftigungsrisiko und die damit verbundene mangelhafte soziale Absicherung sowie die mangelnde Rechtssicherheit vieler Leiharbeitnehmer. Alle drei Risiken überwiegen bei ungelernten Leiharbeitnehmern (siehe im einzelnen Werthebach/Schmidt 1998).

\section{Empirische Untersuchungen zur Leiharbeit}

Angesichts dieser erwarteten Probleme überrascht es, dass es noch wenig gesichertes Wissen zu spezifischen physischen und psychosozialen Belastungen bei Leiharbeit gibt. Neben einer kleinen Anzahl von Monographien zu Hintergründen und Problemen des Einsatzes von Leiharbeitnehmern (z.B. Kock 1990; Kock/Hilse 1989) finden sich einige arbeitsrechtlich fundierte Darstellungen der Chancen und Risiken des Einsatzes von Leiharbeitnehmern aus Arbeitgeber- bzw. gewerkschaftlicher Sicht (z.B. BDA 1987; Frerichs/Krüger 1988; Mayer/ Paasch 1984, 1986) sowie einige Arbeiten aus personalwirtschaftlicher Sicht (z.B. Oltmanns 1979). Auch vom Bundesverband Zeitarbeit sind Untersuchungen zur Entwicklung und ökonomischen Bedeutung von Leiharbeit in Auftrag gegeben worden (z.B. Prognos AG 1974, 1980; BZA 1989). Von der Bundesanstalt für Arbeit (als zentraler Kontrollbehörde des Leiharbeitsmarktes) und dem ihr angeschlossenen Institut für Arbeitsmarkt- und Berufsforschung wurden dagegen in den letzten Jahren keine empirischen Untersuchungen zur Situation im Leiharbeitsgewerbe durchgeführt. Die Bundesanstalt beschränkt sich auf die Erstellung von Statistiken über Art und Umfang der legalen Leiharbeit.

Gesundheitliche und soziale Risiken von Leiharbeit sind zwar bekannt, Informationen zur Häufigkeit und Intensität von Belastungen und Erkrankungen, zu arbeitsbezogenen Konflikten und sozialer Isolation fehlen dagegen. Empirische Arbeiten zu gesundheitlichen Risiken, denen Leiharbeitnehmer verschiedener Gewerke ausgesetzt sind, fehlen ebenfalls. Eine vergleichende Darstellung zur Gesundheit von Leiharbeitnehmern verschiedener Gewerke gegenüber Mitarbeitern aus Stammbelegschaften gleicher Gewerke war gar nicht auszumachen. Unklar ist darum, inwieweit Leiharbeitnehmer stärker gesundheitlich bzw. psychosozial belastet sind als dauerhaft beschäftigte Mitarbeiter gleicher Gewerke und gleichen Alters. Unklar ist ebenfalls, inwieweit soziale und betriebliche Unterstützungsstrukturen (Familie, Freundschaften, Arbeitskollegen, Arbeitnehmervertretung) zur Abpufferung psychosozialer Belastungen zum Tragen kommen. Untersuchungen der Folgen physischer bzw. psychosozialer Belastungen (z.B. Fehlzeiten, Fluktuation) sind in bezug auf Leiharbeitnehmer nicht durchgeführt worden, sondern liegen allein berufsgruppenspezifisch und branchenspezifisch vor (Bundesverband der Betriebskrankenkassen 1992, 1995).

Insgesamt stellt sich die Untersuchungslage zum Thema Leiharbeit als dürftig dar. Sie ist der gesellschaftlichen Relevanz des Themas nicht angemessen. 


\section{$3 \quad$ Entwicklung der Fragestellung}

In der vorliegenden Arbeit wird Rollenklarheit als ein Dreh- und Angelpunkt der Integration von Leiharbeitnehmern untersucht. Begründet werden kann dies mit der Annahme, dass (1) Leiharbeitnehmer aufgrund ihres Status oder aber aufgrund der kurzen Dauer ihrer Betriebszugehörigkeit weniger genau wissen, welche Vorgehensweisen und Leistungen von ihnen erwartet werden, und dass sich (2) diese Unwissenheit in einem - im Vergleich zu festen Mitarbeitern - geringeren Selbstvertrauen niederschlägt.

\section{Rollenklarheit}

Rollenklarheit beschreibt das Ausmaß an Sicherheit, das Personen als Folge eindeutiger und konsistenter Informationen zu verschiedenen Aspekten ihrer Arbeitsrolle erleben (siehe Ilgen/Hollenbeck 1991). Rollenklarheit kann die zu erledigenden Aufgaben betreffen, die hiermit verbundenen Rechte, Pflichten und Verantwortung des Beschäftigten, die Art und Weise der Ausführung der Arbeit (Arbeitsmethoden, zeitlicher Ablauf) sowie die Kriterien, nach denen die resultierenden Leistungen bewertet werden (siehe Ashford/Cummings 1985; Biddle 1979; Breaugh/Colihan 1994; Kahn et al. 1964). Vorgesetzte als auch erfahrene Kollegen können über konsistente und eindeutige Instruktionen und Rückmeldungen unmittelbar zur Klärung des methodischen Vorgehens, der zeitlichen Prioritäten und der Leistungsmaßstäbe beitragen. Nach McGrath (1976) zählt Unsicherheit über Leistungsmaßstäbe zu den am negativsten erlebten Belastungsfaktoren bei der Arbeit.

\section{Bedingungen von Rollenklarheit}

Es ist zu erwarten, dass Beschäftigte mit mehreren Jahren Berufs- bzw. Betriebserfahrung eine ausgeprägte Rollenklarheit entwickelt haben. Sie haben Einsichten und Erfahrungen gewonnen, die sich in Form gezielter Problemfinde- und Problemlösestrategien (Hacker 1992; Krems 1994) sowie einer guten Kenntnis betrieblicher Strukturen, Prozesse und Ansprechpartner zeigen. Berufserfahrenen Leiharbeitnehmern fehlen zwar Detailinformationen, da sich Anlagen und Maschinen, Entscheidungsstrukturen und Produktionsprozesse von Betrieb zu Betrieb unterscheiden. Sie verfügen jedoch in der Regel über Wissensstrukturen, die es ihnen ermöglichen, zu Beginn eines neuen Einsatzes gezielt die entscheidenden Fragen zu stellen und sich innerhalb kurzer Zeit zu orientieren. Ist die Rollenklarheit einzelner Leiharbeitnehmer eher gering ausgeprägt, ist darum erst einmal zu prüfen, inwieweit dies an einer entsprechend geringen Berufserfahrung liegt. Die Rollenklarheit berufsunerfahrener Leiharbeitnehmer dürfte der Rollenklarheit von festen Mitarbeitern mit geringer Berufs- und Betriebserfahrung entsprechen.

Unabhängig von der beruflichen und betrieblichen Erfahrung setzt Rollenklarheit einen zuverlässigen und hinreichenden Austausch aufgabenspezifischer Informationen voraus. Dieser betrifft die technologischen und organisatorischen Erfordernisse, d.h. die Frage, welche Informationen der Beschäftigte in welcher Form zu welchem Zeitpunkt benötigt, um die anstehenden Arbeiten effektiv und effizient planen und erledigen zu können (z.B. 
Arbeitsanweisungen, Bedienungsanleitungen, Leistungsrückmeldung). Er betrifft aber auch die außerfachlichen Anforderungen, also die Frage, welches Verhalten der Beschäftigte im Kollegen- und Vorgesetztenkontakt zeigen und wie er bei der Arbeitsplanung und Problemlösung vorgehen sollte. Ein dritter Aspekt betrifft die Frage, welche Erfahrungen bei der Planung und Erledigung der geforderten Arbeiten anfallen (Praktikerwissen) und auf welche Weise diese Erfahrungen ausgewertet und genutzt werden können.

Von einem reibungslosen Informationsaustausch dürften Leiharbeitnehmer in besonderem Maße profitieren. Leihkräfte sind regelmäßig neu einzuarbeiten, da sie oftmals nur wenige Tage an einem Arbeitsplatz eingesetzt werden. Im einzelnen kann ein Entleihbetrieb die Rollenklarheit der Leiharbeitnehmer dadurch unterstützen, dass er diesen eine Orientierung an Aufgabenbeschreibungen und Leistungsmerkmalen, an betrieblichen Ansprechpartnern, Berichtswegen und Entscheidungsstrukturen ermöglicht. Leistungsrückmeldung ist angeraten bei Arbeits- und Zusammenarbeitsleistungen, die nicht auf Anhieb gelingen können und eine schrittweise Einarbeitung erfordern.

Werden Leiharbeitnehmer bei ihren Arbeitseinsätzen nicht von den Ansprechpartnern und Arbeitsgruppen im Entleihunternehmen akzeptiert (Leiharbeitnehmer sprechen häufig von einem „Klima der Ablehnung“), dann bleiben sie Fremdkörper ohne Anbindung an die Stammbelegschaft. Ohne Anbindung an bestehende Arbeitsgruppen haben Leihkräfte aber kaum Möglichkeiten zur Entwicklung von Rollenklarheit und Arbeitsproduktivität. Der Einsatz von Leihkräften kann dann - aus Sicht der Stammbelegschaft - nicht zum „Erfolgsmodell" werden. Andererseits werden die Leiharbeitnehmer kaum bereit sein, sich für das Entleihunternehmen zu engagieren, wenn sie pauschal abgelehnt werden. Bei der Einarbeitung von Leiharbeitnehmern ist also immer zu prüfen, inwieweit diese von der Stammbelegschaft in die Teamstrukturen einbezogen oder aber ausgegrenzt werden, Rollenunklarheit von Leiharbeitnehmern also ein Statusproblem darstellt. Schlüsselvariablen bei der Untersuchung der Integration von Leiharbeitnehmern sind das Vertrauen (Cook/Wall 1980) und der Zusammenhalt (O’Reilly/Caldwell 1985) zwischen den Leihkräften und den Kollegen der Stammbelegschaft.

\section{Folgen von Rollenklarheit}

Selbstvertrauen beschreibt die Überzeugung eines Menschen, über die wesentlichen Fähigkeiten und Fertigkeiten zu verfügen, ein bestimmtes Ziel selbständig zu erreichen. Vergleicht man zwei gleichermaßen qualifizierte und erfahrene Mitarbeiter, die ein bestimmtes fachliches oder soziales Problem zu lösen haben, dann wird derjenige Mitarbeiter die Problembehebung als belastender erleben, der weniger Vertrauen in seine eigene Leistungsfähigkeit mitbringt, dessen subjektive Ressourcen den kritischen Anforderungen also weniger gut standhalten. Rollenklarheit schafft eine psychologisch sichere Arbeitsumgebung und fördert das fachliche Selbstvertrauen (Werthebach/Kohlisch 1998).

Beschäftigte, die sowohl wissen, was von ihnen erwartet wird, als auch in der Lage sind, diesen Erwartungen nachzukommen, geraten seltener in kritische Situationen, in denen sie Fehler machen, nicht mehr weiter wissen und auf der Rat von Kollegen bzw. Vorgesetzten angewiesen sind. Weniger Mißerfolge bzw. weniger Abhängigkeit von der Expertise anderer Personen führt im Laufe der Zeit dann dazu, dass die Beschäftigten ein gutes Selbstvertrauen entwickeln. Werden Leiharbeiter lange im Unklaren gelassen, nach welchen Vorgaben sie 
sich zu richten haben und worauf es bei der Erledigung ihrer Arbeit ankommt, dann kann sich nur schwerlich ein gutes fachliches Selbstbewußtsein aufbauen.

Je komplexer die betrieblichen Anforderungen und je unübersichtlicher die Probleme und Strukturen, desto wichtiger ist ein kooperatives Arbeitsklima. Insofern wird nicht nur die Rollenklarheit, sondern auch der Zusammenhalt in der Arbeitsgruppe einen direkten Einfluß auf das Selbstvertrauen der Beschäftigten haben. Leiharbeitnehmer dürften aufgrund ihrer Randstellung im Entleihunternehmen in besonderem Maße für negative Bewertungen und Schuldzuweisungen, also ein negatives Arbeitsklima empfänglich sein. Daraus erwächst eine besondere Verantwortung für die Stammbelegschaft und Vorgesetzten im Entleihunternehmen.

Arbeitszufriedenheit beschreibt einen emotionalen Zustand, der aus einer Bewertung der eigenen Arbeitstätigkeit und Arbeitserfahrungen resultiert. Arbeitszufriedenheit stellt sich dann ein, wenn der Beschäftigte seine Ziele erreicht und folglich seine Handlungen als erfolgreich bewerten kann (Kleinbeck 1996). Das Ausmaß der Arbeitszufriedenheit hängt vom Bewertungsmaßstab des Beschäftigten ab. Erwartet der Beschäftigte beispielsweise wenig Informationsaustausch am Arbeitsplatz, dann ist seine Arbeitszufriedenheit größer als bei einem Kollegen, der fehlende Informationen kritisch bewertet und Verbesserungen anmahnt.

Rollenunklarheit beeinträchtigt die Zielerreichung. Wenn Mitarbeiter die Erwartungen ihres Vorgesetzten nicht kennen oder unsicher sind, wie sie ihre Arbeit erledigen sollen, dann ist anzunehmen, dass diese Ungewißheit die Arbeitsleistung beeinträchtigt und folglich zu Unzufriedenheit führt (Sawyer 1992). Obwohl Unterschiede bestehen in dem Ausmaß, in dem Personen fähig sind, Ambiguität zu tolerieren, empfinden Mitarbeiter den Zustand der Ungewißheit in der Regel als belastend. Rollenambiguität geht regelmäßig mit einer geringen Arbeitszufriedenheit einher (Fisher/Gitelson 1983; Jackson/Schuler 1985).

Leiharbeitnehmer dürften aufgrund vergleichsweise kurzer Arbeitseinsätze im Entleihunternehmen geringere Erwartungen an einen qualifikationsgerechten und gesundheitlich unbedenklichen Einsatz sowie einen persönlichen, vertrauensvollen Austausch mit den Kollegen der Stammbelegschaft haben. Folglich ist zu erwarten, dass Leiharbeitnehmer, welche die gleichen Arbeiten zu verrichten haben wie feste Mitarbeiter, mit ihrem Arbeitseinsatz zufriedener sind als die Stammbelegschaft - sofern sie bei den festen Mitarbeitern nicht auf offene Ablehnung stoßen. Bei Leiharbeitnehmern, die unter ungünstigeren Arbeitsbedingungen arbeiten als die Mitarbeiter der Stammbelegschaft, dürfte sich die Benachteiligung nicht in einer entsprechend geringeren Arbeitszufriedenheit niederschlagen, da sich zahlreiche Leiharbeitnehmer in ihren Status gefügt haben und nicht mehr erwarten.

\section{Methodisches Vorgehen}

In der vorliegenden Untersuchung wurde geprüft, inwieweit die Leiharbeitnehmer eines Entleihunternehmens eine vergleichbare Rollenklarheit entwickelt haben wie die festen Mitarbeiter desselben Unternehmens, unter welchen Bedingungen (Informationsaustausch, Kohäsion in der Arbeitsgruppe) Rollenklarheit auftritt und welche Folgen (fachliches Selbstvertrauen, Arbeitszufriedenheit, Gesundheit) mit Rollenklarheit einhergehen.

In die Untersuchung wurden 48 Leiharbeitnehmer eines Unternehmens der chemischen Industrie (Produktionsbereich) einbezogen. Die Leiharbeitnehmer haben zu $90 \%$ eine 
Berufsausbildung absolviert, sind jedoch branchenfremd, d.h. keine Chemiefacharbeiter. Die Vergleichsgruppe besteht aus 85 Arbeitnehmern der Stammbelegschaft desselben Unternehmens, die in kleineren Gruppen tätig sind, aber keine Teamarbeit in reiner Form praktizieren. Der Betrieb ist hierarchisch strukturiert. Jeweils zwei Koordinatoren (ehem. Vorarbeiter) pro Arbeitsgruppe treffen alle Entscheidungen, die sich auf Produktionstermine und -mengen sowie den Personal- und Maschineneinsatz in ihrer Arbeitsgruppe beziehen. Die Arbeitnehmer kontrollieren die Produktqualität sowie ihre Arbeitsgeschwindigkeit. Die Meisterebene tritt nur selten in Erscheinung. In die Vergleichsgruppe sind überwiegend Personen mit abgeschlossener Berufsausbildung einbezogen, jedoch wiederum keine Chemiefacharbeiter. Demographische Daten zur Gruppe der Leiharbeitnehmer und zur Vergleichsgruppe können Tabelle 1 entnommen werden.

In dem hier untersuchten Betrieb werden die Produktionsmaschinen tage- oder wochenweise entweder von festen Mitarbeitern oder von Leihkräften bedient. Leihkräfte machen ca. 25 Prozent der in der Produktion Beschäftigten aus. Sie werden als Springer an Maschinen eingesetzt, an denen ansonsten feste Mitarbeiter arbeiten. Eine Trennung der beiden Personengruppen nach Einsatzbereichen besteht nicht. Aufgrund einer nur kurzen Einarbeitungszeit an der Mehrzahl der Maschinen bietet der von uns untersuchte Produktionsbetrieb gute Voraussetzungen für den Einsatz von Leiharbeitnehmern.

Die 48 Leiharbeitnehmer und 85 Mitarbeiter der Stammbelegschaft wurden innerhalb eines Zeitraums von vier Wochen in schriftlicher Form zu Merkmalen der Arbeit und Zusammenarbeit befragt. Die Befragung erfolgte anonym und auf freiwilliger Basis. Die Befragungszeit wurde als Arbeitszeit vergütet. In den untersuchten Unternehmensbereichen nahmen $94 \%$ der Leiharbeitnehmer und 90 \% der Mitarbeiter der Stammbelegschaft an der Befragung teil.

In Tabelle 2 sind die Skalen vorgestellt, die in der Studie eingesetzt wurden. Die Tabelle ist gegliedert in die Themenbereiche Informationsaustausch, Kohäsion in der Arbeitsgruppe, Rollenklarheit, fachliches Selbstvertrauen, Arbeitszufriedenheit und Gesundheit. Die Skalen wurden bereits in Werthebach, Grote und Schmidkunz (1996) sowie Werthebach, Schmidt und Kleinbeck (1998) eingesetzt, überprüft und dokumentiert. Die Mehrzahl der Skalen sind deutsche Bearbeitungen englischsprachiger Skalen.

\section{Tab. 1: Charakterisierung der untersuchten Personengruppen}

\begin{tabular}{|c|c|c|c|c|c|c|}
\hline Personengruppe & $\begin{array}{c}\text { Alters- } \\
\text { durchschnitt }\end{array}$ & $\begin{array}{c}\text { Frauen- } \\
\text { anteil }\end{array}$ & \begin{tabular}{|c|} 
Hauptschul- \\
abschlu $\beta$
\end{tabular} & $\begin{array}{l}\text { Höhenvertiger } \\
\text { Schulabschluß }\end{array}$ & \begin{tabular}{|l|} 
Abgeschlossene \\
Benufsausbildung
\end{tabular} & \begin{tabular}{|l} 
Durchschnittliche \\
Betriebserfahrung
\end{tabular} \\
\hline Leiharbeitnehmer & $\begin{array}{c}\text { 28 Jahre } \\
\text { Range: }[20 ; 45]\end{array}$ & $7 \%$ & $77 \%$ & $19 \%$ & $90 \%$ & $\begin{array}{c}\text { 1/4 Jahr } \\
\text { Range: }[1 / 12 ; 1 / 2]\end{array}$ \\
\hline Stammbelegschaft & $\begin{array}{c}\text { 39 Jahre } \\
\text { Range: }[23 ; 57]\end{array}$ & $8 \%$ & $84 \%$ & $14 \%$ & $88 \%$ & $\begin{array}{c}\text { 14 Jahre } \\
\text { Range: }[1 ; 30]\end{array}$ \\
\hline
\end{tabular}


Tab. 2: Eingesetzte Untersuchungsinstrumente

\begin{tabular}{|c|c|c|}
\hline Skalenbezeichnung & Quelle & Beispielitem \\
\hline $\begin{array}{l}\text { Informationsver- } \\
\text { sorgung }\end{array}$ & $\begin{array}{l}\text { Werthebach, Schmidt \&Kleinbeck(1998); } \\
\text { Original: Mrvis(1983) }\end{array}$ & $\begin{array}{l}\text { Stehen Ihnen die Informationen, die Sie benötigen, umIhre } \\
\text { Arbeit angemessen zu erledigen, rechtzeitig zur Verfuigung? }\end{array}$ \\
\hline $\begin{array}{l}\text { Leistungsrïickmel- } \\
\text { dung }\end{array}$ & Dto. & $\begin{array}{l}\text { Erfolgt die Rï̈kmeldung zuLeistungen der Gesantgnuppe } \\
\text { [prompt; präżse; konstruktiv]? }\end{array}$ \\
\hline $\begin{array}{l}\text { Kohäsionin der } \\
\text { Arbeitsgnuppe }\end{array}$ & $\begin{array}{l}\text { Werthebach, Grote \&Schmidkunz (1996); } \\
\text { Oiginal: O'Reilly \&Caldwell (1985) }\end{array}$ & $\begin{array}{l}\text { Die Kollegen meiner Arbeitsgnuppe sind bereit, den eimzelnen } \\
\text { gegen Kritik von Außenstehenden zu verteidigen }\end{array}$ \\
\hline $\begin{array}{l}\text { Klarheit der Lei- } \\
\text { stungskriterien }\end{array}$ & $\begin{array}{l}\text { Werthebach, Schmidt \& Kleinbeck(1998); } \\
\text { Oiginal: Breaugh \&Colihan(1994) }\end{array}$ & $\begin{array}{l}\text { Ich weißgenau, welche Arbeitsleistungen meinen unmittelbaren } \\
\text { Vorgesetten zufriedenstellen }\end{array}$ \\
\hline $\begin{array}{l}\text { Klarheit des metho- } \\
\text { dischen Vorgehens }\end{array}$ & Dio. & $\begin{array}{l}\text { Meine Arbeit ist so, daßich immer genau weiß, wie ich bei der } \\
\text { Erledigung meiner Aufgaben vorgehen soll }\end{array}$ \\
\hline $\begin{array}{l}\text { Klarheit der zeitli- } \\
\text { chen Planung }\end{array}$ & Dio. & $\begin{array}{l}\text { Meine Arbeit ist so, daßich immer genau weiß, in welcher } \\
\text { zeitlichen Abfolge ich meine Aufgaben erledigen soll }\end{array}$ \\
\hline $\begin{array}{l}\text { Fachliches Selbst- } \\
\text { vertrauen }\end{array}$ & $\begin{array}{l}\text { Werthebach, Grote \& Schmidkunz (1996) } \\
\text { (Kurzformder Skala); Oiginal: Riggs \& } \\
\text { Knight (1994) }\end{array}$ & $\begin{array}{l}\text { Ich habe Vetrauen in meine Fähigkeiten, meine Arbeit gut zu } \\
\text { schaffen }\end{array}$ \\
\hline $\begin{array}{l}\text { Arbeitsaffieden- } \\
\text { heit }\end{array}$ & $\begin{array}{l}\text { Enzmann \&Kleiber (1989) (Kurzformder } \\
\text { Skala) }\end{array}$ & Ich halte mich germe an meinem Arbeitsplatz auf \\
\hline $\begin{array}{l}\text { Cesundheitliche } \\
\text { Beschwerden }\end{array}$ & $\begin{array}{l}\text { Werthebach(1998a) in Anlehnung an } \\
\text { Zerssen(1975) }\end{array}$ & Herzschmerzen \\
\hline
\end{tabular}

Tab. 3: Eigenschaften der eingesetzten Untersuchungsinstrumente (interne Konsistenz auf der Diagonalen der Matrix) $(\mathbf{N}=$ 133) $(* p<.01)$

\begin{tabular}{|c|c|c|c|c|c|c|c|c|c|c|c|c|c|c|c|}
\hline Variable & $\mathbf{M}$ & SD & \begin{tabular}{|c} 
Bild- \\
bereich \\
\end{tabular} & 1 & 2 & 3 & 4 & 5 & 6 & 7 & 8 & 9 & 10 & Alter & $\mathrm{BE}$ \\
\hline 1. Informationsversorgung & 11.2 & 3.37 & $0-18$ & .84 & & & & & & & & & & & \\
\hline 2. Leistungsrückmeldung & 8.2 & 4.75 & $0-18$ & $.42^{*}$ & .94 & & & & & & & & & & \\
\hline 3. Kohäsion in Arbeitsguppe & 8.0 & 244 & $0-12$ & $.49 *$ & .16 & .89 & & & & & & & & & \\
\hline 4. Klahneit Leistungskiterien & 25.1 & 7.71 & $5-35$ & .19 & $.23^{*}$ & .13 & .89 & & & & & & & & \\
\hline 5. Klarheit method Vorgehen & 29.6 & 5.59 & $5-35$ & $.31^{*}$ & .11 & $.36^{*}$ & $.51 *$ & .87 & & & & & & & \\
\hline 6. Klarheit zeitlichePlanung & 29.4 & 5.32 & $5-35$ & $.26^{*}$ & .13 & .20 & $.48 *$ & $.85^{*}$ & .85 & & & & & & \\
\hline 7. Fachliches Selbstvertrauen & 6.9 & 1.45 & $0-9$ & .18 & .06 & .08 & $.34^{*}$ & $.31^{*}$ & $.43^{*}$ & .73 & & & & & \\
\hline 8. Arbeitsaffiedenheit & 5.6 & 1.83 & $0-9$ & $.32^{*}$ & $.34^{*}$ & $.30^{*}$ & .08 & .01 & -.15 & .04 & .77 & & & & \\
\hline 9. Cesundheitl. Beschwerden & 14.5 & 10.1 & $0-60$ & $-.29 *$ & -.08 & -.12 & -.11 & -.03 & .05 & -.17 & $-.25^{*}$ & .93 & & & \\
\hline 10. Fehlzeiten(nur Stamm) & 4.1 & 296 & $0-14$ & -.20 & $-.24^{*}$ & .10 & .05 & -.02 & -.02 & -.16 & -.07 & .11 & - & & \\
\hline Alter des Beschäftigten & 36.8 & 9.74 & $20-57$ & -.11 & -.06 & .14 & $.32 *$ & $.38^{*}$ & $.35^{*}$ & .21 & -.02 & $.27 *$ & .00 & - & \\
\hline Duver Berufserfahrung(BE) & 11.4 & 9.78 & $0.1-30$ & $-.27 *$ & -.09 & .03 & $.27 *$ & $.23^{*}$ & $.22 *$ & .17 & .09 & $.30^{*}$ & -.01 & $.80^{*}$ & - \\
\hline Duuer Betriebszugehörigkeit & 6.6 & 7.38 & $0.1-28$ & -.21 & -.10 & .12 & .17 & $.29^{*}$ & $.30^{*}$ & .21 & .11 & .19 & .06 & $.56^{*}$ & $.73^{*}$ \\
\hline
\end{tabular}




\section{$5 \quad$ Ergebnisse der Untersuchung}

Die Mittelwerte, Standardabweichungen, Bildbereiche, Interkorrelationen und internen Konsistenzen (Cronbachs alpha) der verwendeten Skalen sind in Tabelle 3 dargestellt. Wie dort ausgewiesen, verfügen die Skalen über zufriedenstellende bis hohe interne Konsistenzen. Bei allen Skalen weisen hohe Werte in der Spalte der Mittelwerte auf eine hohe Variablenausprägung hin. Die Skalen Nummer fünf und sechs sind wie in der Originalarbeit von Breaugh und Colihan (1994) hoch korreliert. Aus praktischen Gründen favorisieren wir jedoch eine getrennte Behandlung der beiden Ambiguitätsfacetten.

\section{Vergleich der Untersuchungsgruppen}

Aufgrund der eingangs geschilderten Problemstellung war zu erwarten, dass Leiharbeitnehmer weniger über betriebliche Vorgänge informiert werden und weniger leistungsbezogene Rückmeldung erhalten als Mitarbeiter der Stammbelegschaft. In dem hier untersuchten Produktionsbetrieb liegen die Verhältnisse jedoch günstiger (siehe Tabelle 4):

- Leiharbeitnehmer werden ebenso gut mit auftragsbezogenen Informationen und Arbeitsmitteln versorgt wie feste Mitarbeiter

- Leiharbeitnehmer erhalten im Durchschnitt mehr Leistungsrückmeldung als feste Mitarbeiter, wenngleich der Unterschied statistisch nicht signifikant ist

- Die befragten Leiharbeitnehmer empfinden in gleichem Maße einen Zusammenhalt unter den Beschäftigten ihrer Arbeitsgruppe wie die festen Mitarbeiter

- Die Rollenklarheit der Gruppe der Leiharbeitnehmer fällt dagegen deutlich hinter der Rollenklarheit der Mitarbeiter der Stammbelegschaft zurück. Die befragten Leiharbeitnehmer kennen sich bei den Leistungskriterien, beim methodischen Vorgehen als auch bei der zeitlichen Ablaufplanung weniger gut aus als die festen Mitarbeiter

- Angesichts der Rollenambiguität der Leihkräfte ist anzunehmen, dass das fachliche Selbstvertrauen und die allgemeine Arbeitszufriedenheit geringer ausgeprägt ist als bei den festen Mitarbeitern. Was das fachliche Selbstvertrauen der Leiharbeitnehmer anbelangt, läßt sich dies feststellen: Leiharbeitnehmer berichten über ein geringeres fachliches Selbstvertrauen als ihre fest eingestellten Kollegen. Die Arbeitszufriedenheit der Leiharbeitnehmer fällt dagegen höher aus als die der festen Mitarbeiter

- Abschließend erhielten die Beschäftigten Fragen zu eventuellen gesundheitlichen Beschwerden. Die befragten Leiharbeitnehmer klagen allgemein über weniger gesundheitliche Beschwerden (Herzschmerzen, Gelenkschmerzen, Konzentrationsschwäche, innere Unruhe, Schlaflosigkeit und Schwindelgefühl) als die festen Mitarbeiter. 
Tab. 4: Vergleich der untersuchten Personengruppen

\begin{tabular}{|l|c|c|c|c|c|}
\hline Variable & $\begin{array}{c}\text { Leiharbeit- } \\
\text { nehmer }\end{array}$ & $\begin{array}{c}\text { Feste Mit- } \\
\text { arbeiter }\end{array}$ & F & df & $\boldsymbol{p}$ \\
\hline \hline 1. Informationsversorgung & 11.8 & 10.7 & 1.92 & $1 ; 132$ & .17 \\
\hline 2. Leistungsrückmeldung & 9.5 & 7.8 & 1.93 & $1 ; 132$ & .17 \\
\hline \hline 3. Kohäsion in Arbeitsgruppe & 8.8 & 8.3 & 1.06 & $1 ; 132$ & .31 \\
\hline \hline 4. Klarheit Leistungskriterien & 22.4 & 26.7 & 9.43 & $1 ; 132$ & .00 \\
\hline 5. Klarheit method. Vorgehen & 26.9 & 31.2 & 19.20 & $1 ; 132$ & .00 \\
\hline 6. Klarheit zeitliche Planung & 26.7 & 31.0 & 20.95 & $1 ; 132$ & .00 \\
\hline \hline 7. Fachliches Selbstvertrauen & 6.4 & 7.1 & 4.27 & $1 ; 132$ & .00 \\
\hline 8. Arbeitszufriedenheit & 6.3 & 5.4 & 4.58 & $1 ; 132$ & .00 \\
\hline \hline 9. Gesundheitl. Beschwerden & 11.0 & 15.9 & 3.89 & $1 ; 132$ & .01 \\
\hline 10. Fehlzeiten & $\begin{array}{l}\text { Leihkräfte werden im Krankheitsfall umgehend er- } \\
\text { setzt }\end{array}$ \\
\hline
\end{tabular}

\section{Bedingungen von Rollenklarheit}

Zur Prüfung der Bedingungen, unter denen Rollenklarheit verstärkt auftritt, wurden hierarchische multiple Regressionen kalkuliert (siehe Tabelle 5). Dabei wurde das Lebensalter und die Berufserfahrung der Beschäftigten sowie die Dauer der Betriebszugehörigkeit in einem ersten Schritt als Kovariate in die Vorhersagegleichung eingeführt. Dabei ist festzustellen, dass mit höherem Alter, mit zunehmender Berufserfahrung bzw. mit längerer Dauer der Betriebszugehörigkeit die Rollenklarheit der Beschäftigten wesentlich stärker ist. Ein reibungsloser arbeitsbezogener Informationsaustausch trägt unabhängig vom Faktor Berufserfahrung zu einer höheren Klarheit bei der Beurteilung von Leistungsergebnissen, im methodischen Vorgehen und bei der zeitlichen Planung der Arbeit bei. Die Kohäsion innerhalb der Arbeitsgruppe und der Arbeitnehmerstatus (Leihkraft vs. fester Mitarbeiter) leisten darüber hinaus keinen wesentlichen eigenständigen Beitrag zur Vorhersage der Rollenklarheit. In der vorliegenden Untersuchung stellt sich die geringere Rollenklarheit der Leiharbeitnehmer also nicht als Statusproblem, sondern als Folge der geringen Berufs- und Betriebserfahrung der Leihkräfte dar. Damit im Einklang steht der Befund, dass die neuen Mitarbeiter der Stammbelegschaft (mit max. zwölf Monaten Betriebszugehörigkeit) eine ebenso geringe Rollenklarheit aufweisen wie die Leiharbeitnehmer $(\mathrm{F}=0.04$ bis 0.19 ; $d f=$ $2 ; 58 ;$ n.s.). 
Tab. 5: Hierarchische multiple Regression zur Vorhersage der Rollenklarheit $(\mathrm{N}=\mathbf{1 3 3})$

\begin{tabular}{|l|c|c|c|c|c|c|c|c|c|c|c|c|c|c|c|c|c|c|}
\hline Kriterium: & \multicolumn{3}{|c|}{ Klarheit der Leistungskriterien } & \multicolumn{3}{c|}{ Klarheit des method. Vorgehens } & \multicolumn{3}{c|}{ Klarheit der zeitlichen Planung } \\
\hline Prädiktoren: & $\mathrm{R}$ & $\mathrm{R}^{2}$ & $\Delta \mathrm{R}^{2}$ & $\mathrm{~F}$ & $\mathrm{df}$ & $p$ & $\mathrm{R}$ & $\mathrm{R}^{2}$ & $\Delta \mathrm{R}^{2}$ & $\mathrm{~F}$ & $\mathrm{df}$ & $p$ & $\mathrm{R}$ & $\mathrm{R}^{2}$ & $\Delta \mathrm{R}^{2}$ & $\mathrm{~F}$ & $\mathrm{df}$ & $p$ \\
\hline $\begin{array}{l}\text { Alter, Berufserfahrung, } \\
\text { Dauer Betriebszugehörigkeit }\end{array}$ & .32 & .11 & - & 4.93 & $\begin{array}{c}3 ; \\
129\end{array}$ & .00 & .45 & .20 & - & 10.59 & $\begin{array}{c}3 ; \\
129\end{array}$ & .00 & .43 & .18 & - & 9.42 & $\begin{array}{c}3 ; \\
129\end{array}$ & .00 \\
\hline $\begin{array}{l}\text { \& Informationsversorgung, } \\
\text { Leistungsrückmeldung }\end{array}$ & .51 & .26 & .15 & 6.82 & $\begin{array}{c}5 ; \\
127\end{array}$ & .00 & .56 & .32 & .12 & 8.98 & $\begin{array}{c}5 ; \\
127\end{array}$ & .00 & .66 & .43 & .25 & 14.60 & $\begin{array}{c}5 ; \\
127\end{array}$ & .00 \\
\hline $\begin{array}{l}\text { \& Kohäsion in der } \\
\text { Arbeitsgruppe }\end{array}$ & .52 & .27 & .01 & 5.80 & $\begin{array}{c}6 ; \\
126\end{array}$ & .00 & .58 & .34 & .02 & 8.07 & $\begin{array}{c}6 \\
126\end{array}$ & .00 & .66 & .43 & .00 & 12.15 & $\begin{array}{c}6 ; \\
126\end{array}$ & .00 \\
\hline $\begin{array}{l}\text { \& Arbeitnehmerstatus } \\
\text { (Stamm vs. Leihkraft) }\end{array}$ & .53 & .28 & .01 & 5.15 & $\begin{array}{c}7 ; \\
125\end{array}$ & .00 & .61 & .37 & .03 & 7.75 & $\begin{array}{c}7 ; \\
125\end{array}$ & .00 & .66 & .44 & .01 & 10.53 & $\begin{array}{c}7 \\
125\end{array}$ & .00 \\
\hline
\end{tabular}

\section{Folgen von Rollenklarheit}

Tabelle 6 bietet eine Übersicht zu den Folgen von Rollenklarheit für das fachliche Selbstvertrauen und die Arbeitszufriedenheit der Beschäftigten. Wie Tabelle 3 bereits zeigt, steht das fachliche Selbstvertrauen in einem deutlich positiven Zusammenhang zur Rollenklarheit: Je stärker die Rollenklarheit, desto größer ist das fachliche Selbstvertrauen der Beschäftigten. Ein Großteil der Kriteriumsvarianz des fachlichen Selbstvertrauens wird jedoch bereits durch die angenommenen Prädiktoren der Rollenklarheit erklärt: Bei gutem Informationsaustausch und in kohäsiven Arbeitsgruppen verfügen die Beschäftigten über ein stärkeres Selbstvertrauen. Abschließend ist zu klären, inwieweit der Arbeitnehmerstatus (Leiharbeiter vs. fester Mitarbeiter) einen eigenständigen Beitrag zur Erklärung des fachlichen Selbstvertrauens aufweist: Es zeigt sich, dass das fachliche Selbstvertrauen zwar mit dem Arbeitnehmerstatus in Zusammenhang steht (siehe Tabelle 4), der entsprechende Teil der Kriteriumsvarianz jedoch bereits von der Rollenklarheit und ihren Prädiktoren aufgeklärt wird. Der Arbeitnehmerstatus leistet also keinen eigenständigen Beitrag zur Erklärung des fachlichen Selbstvertrauens.

Eine starke Rollenklarheit in Verbindung mit einem umfangreichen Informationsaustausch geht mit einer hohen Arbeitszufriedenheit der Beschäftigten einher. Rollenklarheit ohne Berücksichtigung des Informationsaustausches steht dagegen in keiner nennenswerten Beziehung zur Arbeitszufriedenheit der Beschäftigten (siehe Tabelle 3). In der Vorhersagegleichung wirken die Variablen zum Informationsaustausch als Suppressor der Fehlervarianz der Variablen zur Rollenklarheit. Suppressoren binden Fehlervarianz einer anderen Variablen und verhelfen dieser zu einem stärkeren Gewicht in der multiplen Regressionsgleichung. Die inhaltliche Aussage zur Suppressorwirkung lautet, dass (1) Rollenklarheit ohne Informationsaustausch in keiner systematischen Beziehung zur Arbeitszufriedenheit steht und (2) die Kombination aus Informationsaustausch und Rollenklarheit für die Arbeitszufriedenheit wesentlich günstiger ist als ein Informationsaustausch ohne Rollenklarheit. 
Tab. 6: Hierarchische multiple Regression zur Vorhersage des fachlichen Selbstvertrauens und der Arbeitszufriedenheit $(\mathrm{N}=\mathbf{1 3 3})$

\begin{tabular}{|l|c|c|c|c|c|c|c|c|c|c|c|c|}
\hline Kriterium: & \multicolumn{9}{|c|}{ Fachliches Selbstvertrauen } & \multicolumn{5}{|c|}{ Arbeitszufriedenheit } \\
\hline Prädiktoren: & $\mathrm{R}$ & $\mathrm{R}^{2}$ & $\Delta \mathrm{R}^{2}$ & $\mathrm{~F}$ & $\mathrm{df}$ & $p$ & $\mathrm{R}$ & $\mathrm{R}^{2}$ & $\Delta \mathrm{R}^{2}$ & $\mathrm{~F}$ & $\mathrm{df}$ & $p$ \\
\hline $\begin{array}{l}\text { Alter, Berufserfahrung, } \\
\text { Dauer Betriebszugehörigkeit }\end{array}$ & .25 & .06 & - & 2.20 & $\begin{array}{c}3 ; \\
129\end{array}$ & .09 & .18 & .03 & - & 1.10 & $\begin{array}{c}3 ; \\
129\end{array}$ & .35 \\
\hline $\begin{array}{l}\text { \& Informationsversorgung, } \\
\text { Leistungsrückmeldung }\end{array}$ & .49 & .24 & .18 & 6.13 & $\begin{array}{c}5 ; \\
127\end{array}$ & .00 & .47 & .22 & .19 & 5.50 & $\begin{array}{c}5 ; \\
127\end{array}$ & .00 \\
\hline $\begin{array}{l}\text { \& Kohäsion in der } \\
\text { Arbeitsgruppe }\end{array}$ & .61 & .37 & .13 & 9.39 & $\begin{array}{c}6 ; \\
126\end{array}$ & .00 & .51 & .26 & .04 & 5.48 & $\begin{array}{c}6 ; \\
126\end{array}$ & .00 \\
\hline $\begin{array}{l}\text { \& Rollenklarheit (Kriterien, } \\
\text { Vorgehen, zeitl. Planung) }\end{array}$ & .64 & .41 & .04 & 7.02 & $\begin{array}{c}9 ; \\
123\end{array}$ & .00 & .61 & .38 & .12 & 6.02 & $\begin{array}{c}9 ; \\
123\end{array}$ & .00 \\
\hline $\begin{array}{l}\text { \& Arbeitnehmerstatus } \\
\text { Stamm vs. Leihkraft) }\end{array}$ & .64 & .41 & .00 & 6.25 & $\begin{array}{c}10 ; \\
122\end{array}$ & .00 & .64 & .40 & .02 & 6.03 & $\begin{array}{c}10 ; \\
122\end{array}$ & .00 \\
\hline
\end{tabular}

Tab. 7: Hierarchische multiple Regression zur Vorhersage der Gesundheit der Beschäftigten $(\mathrm{N}=133$ bzw. 85)

\begin{tabular}{|l|c|c|c|c|c|c|c|c|c|c|c|c|}
\hline Kriterium: & \multicolumn{3}{|c|}{ Gesundheitliche Beschwerden } & \multicolumn{3}{|c|}{ Fehlzeiten (85 feste Mitarbeiter) } \\
\hline Prädiktoren: & $\mathrm{R}$ & $\mathrm{R}^{2}$ & $\Delta \mathrm{R}^{2}$ & $\mathrm{~F}$ & $\mathrm{df}$ & $\mathrm{p}$ & $\mathrm{R}$ & $\mathrm{R}^{2}$ & $\Delta \mathrm{R}^{2}$ & $\mathrm{~F}$ & $\mathrm{Df}$ & $\mathrm{p}$ \\
\hline $\begin{array}{l}\text { Alter, Berufserfahrung, } \\
\text { Dauer Betriebszugehörigkeit }\end{array}$ & .30 & .09 & - & 3.37 & $\begin{array}{c}3 ; \\
129\end{array}$ & .02 & .13 & .02 & - & 0.59 & $\begin{array}{c}3 ; \\
129\end{array}$ & .62 \\
\hline $\begin{array}{l}\text { \& Informationsversorgung, } \\
\text { Leistungsrückmeldung }\end{array}$ & .38 & .14 & .05 & 3.22 & $\begin{array}{c}5 ; \\
127\end{array}$ & .01 & .46 & .21 & .19 & 4.27 & $\begin{array}{c}5 ; \\
127\end{array}$ & .00 \\
\hline $\begin{array}{l}\text { \& Kohäsion in der } \\
\text { Arbeitsgruppe }\end{array}$ & .38 & .14 & .00 & 2.66 & $\begin{array}{c}6 ; \\
126\end{array}$ & .02 & .50 & .25 & .04 & 4.45 & $\begin{array}{c}6 ; \\
126\end{array}$ & .00 \\
\hline $\begin{array}{l}\text { \& Rollenklarheit (Kriterien, } \\
\text { Vorgehen, zeitl. Planung) }\end{array}$ & .40 & .16 & .02 & 1.91 & $\begin{array}{c}9 ; \\
123\end{array}$ & .06 & .64 & .41 & .16 & 5.63 & $\begin{array}{c}9 ; \\
123\end{array}$ & .00 \\
\hline $\begin{array}{l}\text { \& Selbstvertrauen, } \\
\text { Arbeitszufriedenheit }\end{array}$ & .45 & .20 & .04 & 1.98 & $\begin{array}{c}11 ; \\
121\end{array}$ & .04 & .65 & .42 & .01 & 4.54 & $\begin{array}{c}11 ; \\
121\end{array}$ & .00 \\
\hline $\begin{array}{l}\text { \& Arbeitnehmerstatus } \\
\text { Stamm vs. Leihkraft) }\end{array}$ & .49 & .24 & .04 & 2.28 & $\begin{array}{c}12 ; \\
120\end{array}$ & .01 & $\begin{array}{c}\text { Leihkräfte werden im Krankheits- } \\
\text { fall umgehend ersetzt }\end{array}$ \\
\hline
\end{tabular}

Tabelle 7 bietet eine Übersicht zu den Folgen von Rollenklarheit für die Gesundheit der Beschäftigten. Gesundheitliche Beschwerden stehen in keinem bedeutsamen Zusammenhang mit der Rollenklarheit der Beschäftigten. Ein schlechter Informationsaustausch sowie eine geringe Arbeitszufriedenheit stehen dagegen mit der Anzahl und Häufigkeit gesundheitlicher Beschwerden in Zusammenhang. Wie zu erwarten ist, sind gesundheitliche Beschwerden mit höherem Alter zahlreicher bzw. häufiger. Mit dem Alter bzw. der Dauer der Betriebszugehörigkeit korreliert vor allem die Häufigkeit von Herzschmerzen $(\mathrm{r}=.38 \mathrm{bzw}$. $\mathrm{r}=.43 ; \mathrm{df}=131 ; p<.001)$.

In Arbeitsgruppen, in denen ein guter Informationsaustausch besteht, fehlen die Mitarbeiter der Stammbelegschaft deutlich seltener als in Arbeitsgruppen, in denen es am Informationsaustausch mangelt. Eine starke Rollenklarheit geht nur in Verbindung mit einem umfangreichen Informationsaustausch mit wenigen Fehlzeiten der dort Beschäftigten einher. Rollen- 
klarheit ohne Berücksichtigung des Informationsaustausches steht in keiner Beziehung zu den Fehlzeiten der Beschäftigten (siehe Tabelle 3). In der Vorhersagegleichung wirken die Variablen zum Informationsaustausch (wie beim Kriterium Arbeitszufriedenheit) als Suppressor der Fehlervarianz der Variablen zur Rollenklarheit. Interessanterweise stehen die Fehlzeiten der Beschäftigten in keiner nennenswerten statistischen Beziehung zu den berichteten gesundheitlichen Beschwerden. Über Leiharbeitnehmer kann an dieser Stelle keine Aussage getroffen werden, da diese im Krankheitsfall umgehend ausgetauscht werden.

\section{Diskussion}

In der vorliegenden Untersuchung ließ sich feststellen, dass die Leiharbeitnehmer vergleichbaren Arbeitsbedingungen ausgesetzt sind wie die festen Mitarbeiter. Die Leihkräfte werden tage- bzw. wochenweise als Springer an Maschinen eingesetzt, an denen in der übrigen Zeit des Jahres feste Mitarbeiter arbeiten. Darüber hinaus werden die Leihkräfte mindestens ebenso gut instruiert, mit Arbeitsmitteln versorgt und mit Leistungsrückmeldung versehen wie die festen Mitarbeiter im Entleihunternehmen. Während die festen Mitarbeiter gut eingearbeitet und zur Fehlervermeidung weniger stark auf Leistungsrückmeldung angewiesen sind, erhalten Leihkräfte individuell zugeschnittene Rückmeldung zur Verbesserung ihrer Maschinenbedienung.

Dennoch ist die Rollenklarheit der Leiharbeitnehmer deutlich geringer als die der festen Mitarbeiter. Dabei ist zu bedenken, dass die Entwicklung von Rollenklarheit (insbesondere die Klarheit der Leistungskriterien) eine beträchtliche Dauer der Betriebszugehörigkeit und eine kontinuierliche Reflexion der eigenen Arbeitsleistung erfordert. Dies läßt sich vor allem daran erkennen, dass neue Mitarbeiter der Stammbelegschaft (mit max. zwölf Monaten Betriebszugehörigkeit) über eine ebenso geringe Rollenklarheit verfügen wie die Leiharbeitnehmer. Der Befund verdeutlicht, dass im hier untersuchten Betrieb die vergleichsweise geringe Rollenklarheit der Leiharbeitnehmer weniger ein Statusproblem als vielmehr ein Problem der kurzen Betriebszugehörigkeit der Leihkräfte ist. Die vergleichsweise positive Beurteilung der Gruppenkohäsion durch die Leiharbeitnehmer deutet ebenfalls darauf hin, dass sich die Leiharbeitnehmer von den festen Mitarbeitern nicht ausgegrenzt fühlen, die Leiharbeitnehmer im hier untersuchten Unternehmen also kein gravierendes Statusproblem haben.

Mit einer geringen Rollenklarheit geht ein geringes fachliches Selbstvertrauen einher. Setzt man voraus, dass die Höhe des fachlichen Selbstvertrauens die Arbeitsleistung und Gesundheit der Beschäftigten mitbestimmt, dann besteht Handlungsbedarf in Zusammenhang mit der vergleichsweise geringen Rollenklarheit der Leihkräfte und neuen festen Mitarbeiter. Tabelle 3 zeigt, dass vor allem eine reibungslose Informationsversorgung der Beschäftigten mit einer hohen Rollenklarheit einher geht. Daraus folgt, dass Maßnahmen zur Einarbeitung neuer Mitarbeiter bzw. zur Teamentwicklung, in denen die Verantwortung jedes Beschäftigten für den Erfolg der Arbeitsgruppe und des Unternehmens reflektiert wird, einen günstigen Einfluß auf die Rollenklarheit und das fachliche Selbstvertrauen neuer Mitarbeiter haben können.

Die Arbeitszufriedenheit der Leiharbeitnehmer ist deutlich stärker ausgeprägt als die der festen Mitarbeiter. Dieser Befund läßt sich mit den wechselnden Einsatzstellen im Betrieb und der damit verbundenen Abwechslung sowie mit dem Wunsch und der Hoffnung auf eine 
Übernahme durch das Entleihunternehmen erklären. Überhaupt dürfte für die Leiharbeitnehmer ein Anreiz bestehen, sich über gute Leistungen für eine Übernahme zu empfehlen und als Springer in einer Gruppe fester Mitarbeiter nicht aufgrund mangelhafter Leistungen unter Druck zu geraten. Signalisiert ein Entleihunternehmen, dass es die Absicht hat, qualifizierte und gut motivierte Leiharbeitnehmer als feste Mitarbeiter zu übernehmen, dann hat dies zwei Effekte: Zum einen erhalten erfolgszuversichtliche Leihkräfte einen „Motivationsschub“, zum anderen stellt das Unternehmen klar, dass entsprechende Leiharbeitnehmer wertvolle Arbeitskräfte sind, die eine sorgfältige Einarbeitung und gute Behandlung verdienen.

Die Gruppe der Leiharbeitnehmer klagt über weniger gesundheitliche Beschwerden als die festen Mitarbeiter. Möglicherweise beugt der häufige Arbeitsplatzwechsel der Leiharbeitnehmer einer höheren Beanspruchung durch physikalische, chemische und organisatorische Arbeitsbedingungen vor. Beispielsweise könnten die gesundheitlichen Beschwerden der festen Mitarbeiter mit einem erhöhten Erleben von Verantwortungsdruck einher gehen. Eine zweite Erklärung wäre, dass die Leiharbeitnehmer aus anderen Betrieben höhere Arbeitsbelastungen kennen und die quantitative Belastung im untersuchten Entleihunternehmen als weniger stark bewerten (,herunterspielen“). Und drittens darf nicht vernachlässigt werden, dass die hier untersuchten Leiharbeitnehmer im Durchschnitt etwa 11 Jahre jünger sind als die festen Mitarbeiter. Dies verweist auf den allgemeinen Befund, dass die Beschäftigten mit zunehmendem Alter sowohl kritischer gegenüber organisatorischen Problemen als auch gesundheitlich anfälliger werden. Welche der Erklärungen die zutreffende ist, kann aufgrund der vorliegenden Befunde nicht geklärt werden. Darum sollten Ursachenfaktoren gesundheitlicher Beschwerden in nachfolgenden Untersuchungen ausführlicher untersucht werden. Fehlzeiten stehen nicht mit dem Alter oder den gesundheitlichen Beschwerden der Beschäftigten in Zusammenhang. Deutlich wurde dagegen, dass die Mitarbeiter der Stammbelegschaft in Arbeitsgruppen mit einem guten Informationsaustausch deutlich seltener fehlen als in Arbeitsgruppen mit schlechtem Informationsaustausch. Insbesondere eine systematische Leistungsrückmeldung an die Arbeitsgruppe scheint dem Auftreten von Fehlzeiten entgegenzuwirken. Darüber hinaus zeigte sich, dass eine starke Rollenklarheit nur in Verbindung mit einem umfangreichen Informationsaustausch mit wenigen Fehlzeiten der dort Beschäftigten einher geht. Da Leiharbeitnehmer im Krankheitsfall umgehend ausgetauscht werden, beziehen sich Befunde zu Fehlzeiten ausschließlich auf Mitarbeiter der Stammbelegschaft. Die hier vorgestellte Untersuchung ist weit davon entfernt, die Situation der Leiharbeitnehmer in Deutschland repräsentativ wiederzugeben. Dies war allerdings auch nicht beabsichtigt. Vielmehr ging es darum, die Arbeitssituation in einem Betrieb, der in größerem Umfang Leiharbeitnehmer mit einem eng umschriebenen Qualifikationsprofil (im vorliegenden Fall: branchenfremde Facharbeiter) einsetzt, präzise zu erfassen und einen Ansatzpunkt zur Integration von Leiharbeitnehmern (Rollenklarheit) genauer zu prüfen. Das hier vorgestellte Untersuchungsinstrument dürfte aber aufgrund seiner guten metrischen Eigenschaften geeignet zu sein, auch in einer Repräsentativuntersuchung eingesetzt zu werden.

\section{Literatur}

Ashford, Susan J., L.L. Cummings (1985): Proactive feedback seeking: The instrumental use of the information environment; in: Journal of Occupational Psychology, 58, 67-79. 
Breaugh, James A., John P. Colihan (1994): Measuring facets of job ambiguity: Construct validity evidence; in: Journal of Applied Psychology, 79, 191-202.

Bundesverband der Betriebskrankenkassen (Hg.) (1992/95): Betriebsklima: Ergebnisse einer Repräsentativerhebung. Januar 1992 und 1995.

Bundesverband Zeitarbeit e.V. (Hrsg.) (1989): Zeitarbeit: Im Spiegel der Meinungsforschung. Bonn. Bundesvereinigung der deutschen Arbeitgeberverbände (BDA) (Hrsg.) (1987): Arbeitnehmerüberlassung oder Werkvertrag - Möglichkeiten und Risiken des Fremdpersonaleinsatzes in Betrieben. Dokumentation der Fachtagung der BDA, Köln.

Cook, John, Toby Wall (1980): New work attitude measures of trust, organizational commitment and personal need non-fulfilment; in: Journal of Occupational Psychology, 53, 39-52.

Enzmann, Dirk, Dieter Kleiber (1989): Helfer-Leiden. Stress und Burnout in psychosozialen Berufen. Heidelberg: Asanger.

Fisher, C.D., Richard Gitelson (1983): A meta-analysis of the correlates of role conflict and ambiguity; in: Journal of Applied Psychology, 68, 320-333.

Frerichs, Joke, M. Krüger (1988): Beschäftigung ganz unten - Leiharbeit in NRW (Hefte zur Arbeitsmarktpolitik Nr.1). Düsseldorf.

Hacker, Winfried (1992): Expertenkönnen: Erkennen und Vermitteln. Göttingen/Stuttgart.

Ilgen, Daniel R., John R. Hollenbeck (1991): The structure of work: Job design and roles; in: Marvin

D. Dunnette \& L.M. Hough (Eds.): Handbook of Industrial and Organizational Psychology (Vol. 2, pp. 165-207). Palo Alto, CA.

Jackson, S.E., R.S. Schuler (1985): A meta-analysis and conceptual critique of research on role ambiguity and role conflict in work settings; in: Organizational Behavior and Human Decision Processes, 36, 16-78.

Kahn, Robert L., Donald M. Wolfe, Robert P. Quinn, J. Diedrick Snoek, Robert A. Rosenthal (1964): Organizational Stress: Studies in Role Conflict and Ambiguity. New York.

Kleinbeck, Uwe Erich (1996): Arbeitsmotivation. Entstehung, Wirkung und Förderung. Weinheim/ München.

Kock, Klaus (1990): Die austauschbare Belegschaft: Flexibilisierung durch Fremdfirmen und Leiharbeit. Köln.

Kock, Klaus, Horst Hilse (1989): Beschäftigungsförderung durch Leiharbeit? Düsseldorf.

Krems, Josef F. (1994): Wissensbasierte Urteilsbildung. Diagnostisches Problemlösen durch Experten und Expertensysteme. Bern.

Mayer, Udo, Ulrich Paasch (1984): Zur Praxis illegaler Beschäftigung im Baugewerbe. Zwischenbericht zum Projekt 'Leiharbeit im Baugewerbe' an der Hochschule für Wirtschaft \& Politik, Hamburg. Düsseldorf.

Mayer, Udo, Ulrich Paasch (1986): Arbeitnehmer 2. Klasse. Düsseldorf.

McGrath, J.E. (1976). Stress and behavior in organizations; in: Marvin D. Dunnette (Ed.): Handbook of Industrial and Organizational Psychology. New York, 1351-1395.

Mirvis, Philip H. (1983) : Assessing the process and progress of change in organizational change programs; in: Stanley E. Seashore, Edward E. Lawler III, Philip H. Mirvis, Cortlandt Cammann (Eds.): Assessing Organizational Change. A Guide to Methods, Measures, and Practices. New York, 417-451.

Oltmanns, Christoph (1979): Personalleasing: Personaleinsatz-, Personalbedarfs- und Personalstrukturplanung unter besonderer Berücksichtigung des Personalleasings. Wiesbaden.

O'Reilly, Charles A. III, Caldwell, D.F. (1985): The impact of normative social influence and cohesiveness on task perceptions and attitudes: A social information processing approach; in: Journal of Occupational Psychology, 58, 193-206.

Prognos AG (Hrsg.) (1974): Zeitarbeit. Einzel- und gesamtwirtschaftliche Aspekte im Urteil von sachverständigen Befragten. Basel.

Prognos AG (Hrsg.) (1980). Zeitarbeit II - Schlußbericht. Untersuchung erarbeitet im Auftrag des Bundesverbandes Zeitarbeit (BZA). Basel. 
Sader, Manfred (1991): Psychologie der Gruppe (2. Aufl.). Weinheim / München.

Sawyer, John E. (1992): Goal and process clarity: Specification of multiple constructs of role ambiguity and a structural equation model of their antecedents and consequences; in: Journal of Applied Psychology, 77, 130-142.

Werthebach, Markus, Klaudia Grote, Heinz Schmidkunz (1996): Berufliche und betriebliche Sozialisation: Ein Modellversuch zur berufsfeldübergreifenden und fallbezogenen Ausbildung von Chemikanten / Papiermachern, Industriemechanikern und Prozeßleitelektronikern / Energieelektronikern; in: Zeitschrift für Klientenzentrierung, 1, 73-121.

Werthebach, Markus, Olaf Kohlisch (1998): Einsatz einer verfahrenstechnischen Simulation in der Ausbildung von Chemikanten, Industriemechanikern und Prozeßleitelektronikern; in: Zeitschrift für Klientenzentrierung, 3, 39-83.

Werthebach, Markus, Klaus-Helmut Schmidt (1998): Leiharbeit - Risiken und Perspektiven aus arbeitsund organisationspsychologischer Sicht; in: Zeitschrift für Klientenzentrierung, 3, 1-38.

Werthebach, Markus, Klaus-Helmut Schmidt, Uwe Kleinbeck (1998): Produktivitätsförderung in der Personalverwaltung durch Einführung eines Partizipativen Produktivitätsmanagements (PPM); in: Zeitschrift für Arbeits- und Organisationspsychologie, 42, 100-108.

Zerssen, D. von (1975): Die Beschwerden-Liste. Manual. Weinheim.

Anschrift der Verfasser:

Markus Werthebach, Dipl.-Kfm., Dipl.-Psych.

Gesellschaft für Managementberatung und Arbeitsgestaltung GbR

Dortmunder Straße 13

44536 Lünen

Daniel Sodenkamp, Dipl.-Psych.

Universität Dortmund, Fachbereich 14

Zusatzstudiengang Organisationspsychologie

Emil-Figge-Straße 50

44221 Dortmund

Klaus-Helmut Schmidt, Dipl.-Psych., Dr. phil., PD

Institut für Arbeitsphysiologie an der Universität Dortmund

Ardeystraße 67

44139 Dortmund 